\section{Endodontics Manual for the General Dentist}

Martin Trope, D.M.D.; Gilberto Debelian, D.M.D., Ph.D. London: Quintessence Publishing Co, Ltd., 2006; www.quintpub. co.uk.

84 pp. Illus., indexed.

$\$ 68.00$ softcover

ISBN 1-85097-089-0

Reviewed by:

Patricia Bauer, D.D.S., M.S.

Dr. Bauer is Associate Clinical Professor, Department of Cariology, Restorative Sciences, and Endodontics, University of Michigan, School of Dentistry, 1011 N. University, Ann Arbor, MI 48109-1078; 734-936-3329 phone; 734-936-1597 fax; taba@ umich.edu.
The purpose of this endodontics manual is to provide scientific and clinical data to aid the practitioner in arriving at a correct diagnosis and to offer a treatment regimen based on biological fundamentals governing a successful outcome. New materials and techniques are presented.

Major points of interest include a biological review of the presence and progression of pulpal disease and summary of tests and findings necessary in formulating a diagnosis. The review was concise yet thorough and served to simplify the multifaceted diagnostic dilemma. In addition, the final chapter introduces an endodontic case difficulty assessment form developed by the American Association of Endodontists. Using it enables a practitioner to assign a level of difficulty to a particular case, providing concrete guidance in choosing cases commensurate with the experience and comfort of the clinician.

A strength of this manual lies in its ability to consistently relate the scientific, biological concept to the clinical process so that a successful outcome is accomplished.

It is not simply a "how to" but a "why" and supplies the reader with clear and relevant literature citations. The passages on instruments and cleaning/shaping are well written and complete, but those on root canal filling are too limited. A weakness lies in the presentation of a single technique, based on a filling material developed by one of the authors. A broader presentation on obturation could have been discussed.

The manner in which the subject matter is presented is straightforward and organized. Content is a balanced mix of science and technique supported by literature. Chapter 1, an "Introduction to Endodontics," presents vital and necrotic pulp therapy. Chapter 2 discusses diagnosis, and Chapters 3 and 4 present treatment success and the clinical process of root canal therapy. In the final chapter, treatment outcome and prognosis are addressed. Throughout the chapters are numerous photographs and diagrams that enrich reading and clarify the information and explanations. The photographs are exceptional in quality and descriptive nature and enhance the written text.

This manual clearly demonstrates a logical and practical approach to achieve a correct endodontic diagnosis and realize a successful root canal outcome. While the material on filling is narrow and reflects the treatment protocol of the authors, I would recommend the manual for a student or general practitioner in pursuit of extended knowledge or potential solutions to problems they may have encountered in the accomplishment of root canal procedures. It could be useful as a reference manual in a dental/medical library as well. 